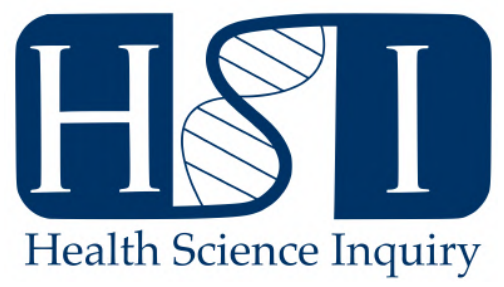

\title{
Antifragile by Design Using Antifragility as a Guiding Principle in Future Rural eHealth Implementation \& Evaluation
}

\author{
Samuel Petrie ${ }^{1}$, Dr. Paul Peters ${ }^{1}$, and Dr. Dean Carson ${ }^{2}$ \\ ${ }^{1}$ Spatial Determinants of Health Lab, Department of Health Sciences, Carleton University \\ ${ }^{2}$ Department of Epidemiology and Global Health, Umeå University, Sweden
}

\section{INTRODUCTION}

Antifragility as a concept was first introduced in the 2012 book Antifragile by Nicholas Taleb [1]. Taleb posits that an antifragile unit stands to gain, rather than be harmed from volatility [1]. Examples of antifragility include bones becoming stronger from small stressors, our immune system building strength from fighting off viruses, and muscle mass being built through continuous use. This analogy can be extended to rural eHealth ${ }^{1}$ interventions, where small failures would strengthen the system as it learned from past mistakes, rather than making it weaker and leading to overall failure. The future of health services delivery in rural communities could depend on being able to effectively implement eHealth interventions and see them scale-up to regional or national initiatives.

Antifragility has been used outside health as a guiding concept for research: including transit planning in Australia [2], risk analysis [3], and systems engineering [4]. In health there are fewer examples, but it can be seen in the implementation of a Swedish Virtual Health Room (or VHR); a community-based rural eHealth intervention [5]. The room is located in a school in the village of Slussfors and contains various sophisticated technologies to monitor health needs of residents. These include thermometers, glucometers, blood-pressure cuffs, heart rate monitors, and video-conferencing technology for consultations. It has been effectively introduced where initiatives in similar contexts have failed, and it has increased the accessibility to health services for rural residents in the village and surrounding area. In Canada, antifragile implementation of online health portals could learn from Quebec's 'carnet santé' service, which has thrived, in comparison to the MyHealthNS portal in Nova Scotia which has declined.

${ }^{1} \mathrm{eHealth}$, for the purpose of this article, is defined as any electronic medium used to administer health services

\section{Exploring ANTIFRAGILITy THROUgh THE Virtual Health Room Case Study}

The VHR intervention in Sweden exemplifies the importance of optionality, non-linear evaluation, and starting small in project design. Optionality is defined as the character of the option: or simply the ability to make choices [1]. Fragile projects lack optionality, while antifragile projects provide an abundance of it. Optionality requires input from both sides of the patient-provider dyad. Ultimately eHealth initiatives should use a patient centered approach [6], putting them at the heart of their model. The Swedish VHR provided optionality by ensuring video cameras and televisions could be used for things other than health - such as education, psychological services, or secure meetings.

Non-linear evaluation refers to the process of diligently considering upstream and downstream changes to systems following an intervention's implementation [7]. It forgoes labeling a project as a 'success' or 'failure', and instead seeks to characterize barriers and limitations, and continually identify solutions to them. Inherent in implementation processes are consequences which cannot be predicted. As such, fragile projects will miss key changes in the system by approaching evaluation linearly. Antifragile projects embrace a holistic evaluation approach, highlighting prominent interdependencies through multiple levels within systems. The Swedish VHR team achieved this by evaluating the VHR non-linearly, through multiple feedback streams and checkpoints from a diverse range of stakeholders. This includes patients, providers, policy-makers, and international collaborators. Barriers were addressed in real-time and seen to strengthen the overall system.

Starting small refers to the iterative process of project design, wherein research teams should begin with appropriately scaled pilot projects, before rolling out more comprehensive initiatives [7]. It avoids path-dependence, which locks implementation teams into following difficult methods of program execution due to considerable investment of time and/or money by starting large [8]. The VHR in the Swedish case started small, with multiple rounds of pa- 
tient and provider feedback, before slowly introducing the technology outlined previously into the room, allowing the research team time to tinker and iterate.

\section{ApPlying AntifRagility in a CAnAdian CONTEXT}

Online health portals in Canada could be important for rural communities to increase access to health services. Quebec has embraced antifragile project design during their implementation in ways other provinces (namely Nova Scotia's MyHealthNS portal) has not. Quebec's carnet santé service allows patients to register for themselves, or have their caregiver or physician sign them up, an example of $o p$ tionality [9]. The MyHealthNS portal requires physicians to register their patients resulting in significantly lower numbers enrolling [10].

Using non-linear evaluation, the carnet santé service had perspectives from a diverse range of stakeholders in their implementation process, while the MyHealthNS portal used a top-down model. The result, again, was many more physicians and patients enrolling in Quebec's portal than Nova Scotia's. Lastly, Quebec slowly introduced services to their portal - starting with receiving bloodwork, to now being able to view x-ray results [9]. Nova Scotia attempted to introduce everything at once, confusing both patients and providers [10]. If a pan-Canadian portal is to be introduced, implementors would be wise to consider the success of Quebec's 'carnet santé' and Sweden's VHRs as an example of the potential of antifragile project design.

\section{Conclusion}

Evidence and theory suggest that consciously applying antifragile thinking starting in the project design phase will result in greater numbers of projects being able to thrive. These thriving projects would be characterized as being enthusiastically supported by practitioners, used by a diversity of stakeholders, with frequent and positive engagement by patients or clients, movement from a 'pilot' to part of routine health services framework, a secure funding source(s) identified, and the potential to 'scale-up' services and expand to other locations.

\section{REFERENCES}

[1] Taleb NN. Antifragile: Things that gain from disorder. vol. 3. Random House Incorporated; 2012.
[2] Isted R. The use of antifragility heuristics in transport planning. In: Australian Institute of Traffic Planning and Management (AITPM) National Conference, 2014, Adelaide, South Australia, Australia. 3; 2014 . .

[3] Aven T. The concept of antifragility and its implications for the practice of risk analysis. Risk analysis. 2015;35(3):476-483.

[4] Verhulsta E. Applying systems and safety engineering principles for antifragility. Procedia computer science. 2014;32:842-849.

[5] Hurtig AK. Strengthening community-based health systems through e-health innovations?;. Available from: https://www.umu.se/en/research/projects/ strengthening-community-based-health-systemsthrough-e-health-innovations/.

[6] Townsend A, Leese J, Adam P, McDonald M, Li LC, Kerr S, et al. eHealth, participatory medicine, and ethical care: a focus group study of patients' and health care providers' use of health-related internet information. Journal of medical Internet research. 2015;17(6):e155.

[7] Braithwaite J, Churruca K, Ellis LA, c Long J, ClayWilliams R, Damen N, et al. Complexity science in healthcare-aspirations, approaches, applications and accomplishments: a white paper. Macquarie University; 2017.

[8] Flyvbjerg B. Big is Fragile: An attempt at theorizing scale. by B Flyvbjerg Oxford: Oxford University Press (2016b) "The fallacy of beneficial ignorance: A test of hirschman's hiding hand" In: World Development. 2016;84:176-189.

[9] Days of Past Futures. Coming Tuesday in Quebec: Your digital health file at your fingertips;. Available from: https://prich5757. wordpress.com/2018/ 11/16/myhealthns-a-perplexing-patient-portalposer/.

[10] Fidelman C. MyHealthNS - A perplexing patient portal poser;. Available from: https: //montrealgazette.com/news/local-news/comingtuesday-in-quebec-your-digital-health-fileat-your-fingertips. 

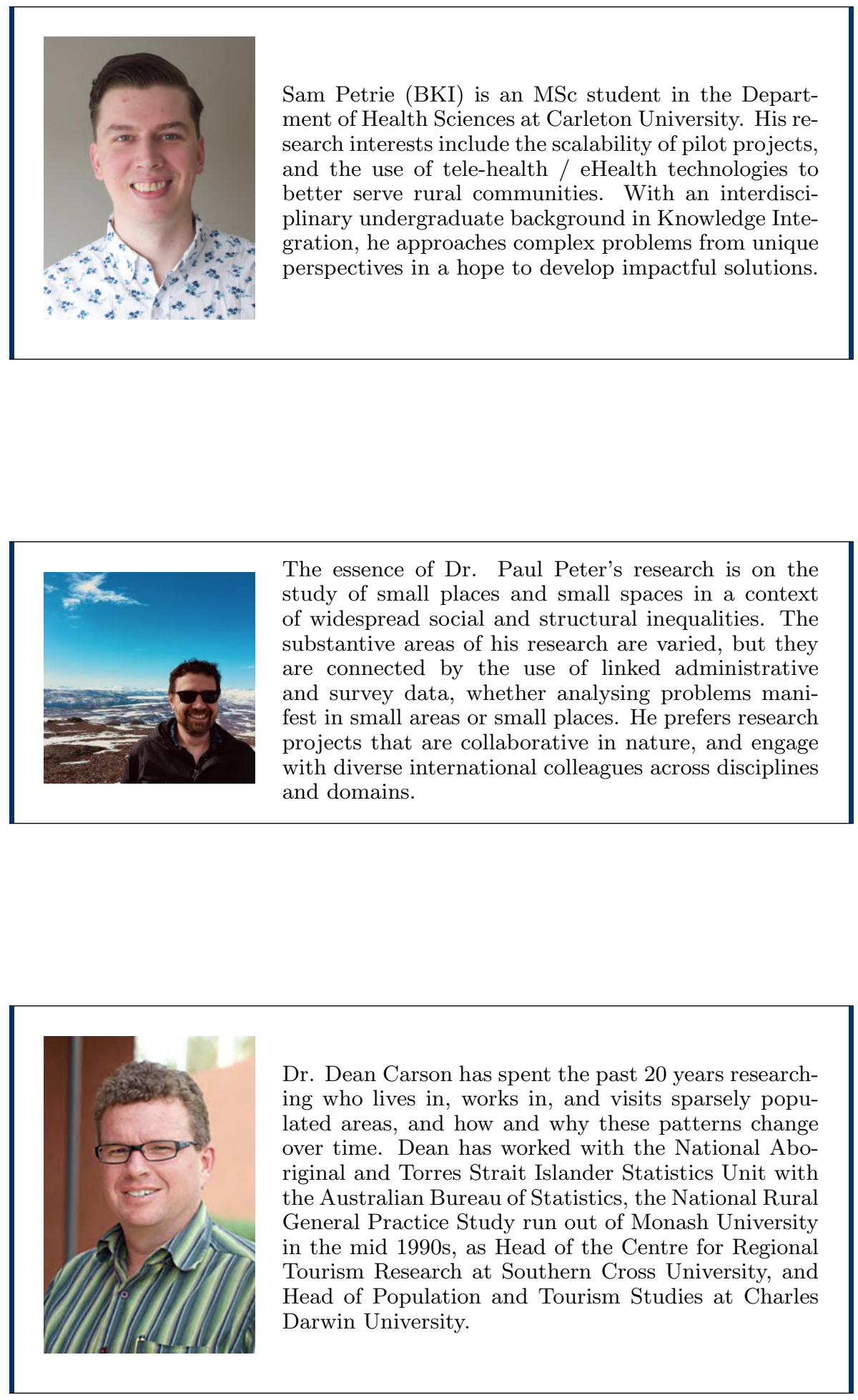